\title{
Weighting Factor Design in Model Predictive Control for Power Converters
}

\author{
Ricardo Enrique Pérez-Guzmán, Marco Rivera, and Patrick W. Wheeler
}

\begin{abstract}
Model-based predictive control is an important multi-objective control strategy in power converters, but it must be properly designed. This research shows different alternatives to improve the cost function in model-based predictive control. For this purpose, the procedure for adjusting the weight factors in the cost functions, all relevant elements and some examples that demonstrate the influence of each term in the equation is presented. The cost functions are characterized and simulated to verify the effectiveness of the working method and the claims made in this investigation. It was found that modelbased predictive control is a powerful alternative when there are multiple objectives, which can be included efficiently within a cost function. However, it is necessary to investigate the ideal value of each of these terms.
\end{abstract}

Keywords-Predictive control; cost function; weighting factor.

\section{INTRODUCCIÓN}

En los últimos años, el control predictivo basado en modelo (MPC) se ha propuesto como una alternativa interesante para el control en convertidores en potencia. Algunos de sus beneficios potenciales radican en la aplicación de sistemas multivariables y la obtención de una respuesta dinámica rápida [1]. Actualmente, los enfoques de MPC se pueden encontrar en la literatura para casi todas las aplicaciones en electrónica de potencia [2]. La razón principal es que el poder computacional de los microprocesadores modernos ha aumentado considerablemente, permitiendo implementar estrategias de control más complejas e inteligentes [3], [4].

Debido a la amplia gama de estrategias de control predictivo basado en modelos, las técnicas aplicadas a estos se han clasificado en dos categorías principales: MPC de estado contínuo (CCS-MPC) y MPC de estado finito (FCS-MPC) [5]. En el primer tipo [6], la variable de control es el voltaje de salida del convertidor, y la solución propone resolver un problema de optimización para predecir el horizonte de estados, en cada intervalo de muestreo. Este comportamiento se aplica generalmente mediante la modulación por ancho de pulso (PWM), o la modulación vectorial espacial (SVPWM) [5]. El segundo tipo de estrategia control (FCS-MPC), utiliza la naturaleza discreta del convertidor para resolver el problema de optimización, utilizando una función de costo única. Aquí, la entrada está restringida a un conjunto finito de valores

Ricardo Enrique Pérez-Guzmán, (Doctorado en Sistemas de Ingeniería), Departamento de Ciencias de la Computación, Facultad de Ingeniería, Universidad de Talca, Curicó, Chile (e-mail: riperez@utalca.cl).

Marco Rivera, Centro Tecnológico de Conversión de Energías, Facultad de Ingeniería, Universidad de Talca, Curicó, Chile (e-mail: marcoriv@utalca.cl).

Patrick W. Wheeler, Faculty of Science and Engineering, Department of Electrical and Electronic Engineering, University of Nottingham, Nottingham NG7 2RD U.K. (e-mail: pat.wheeler@ nottingham.ac.uk). discretos [7] y el modelo del sistema se evalúa para cada secuencia de posibilidades y luego se compara con la referencia, para seleccionar el mejor vector de voltaje [8].

Las principales ventajas del MPC son el control de manera conjunta de los objetivos, las variables y las restricciones, en una sola función de costo. De esta manera, las variables típicas como la corriente, el voltaje, el torque, o el flujo, pueden ser controlados mientras se mantienen algunos requisitos de control adicionales, como la reducción de la frecuencia de conmutación, la reducción de voltaje en modo común, o el control de la potencia reactiva [9].

No obstante, incluir más de una variable dentro de la función de costo incrementa considerablemete la complejidad de la estrategia de control, sobre todo si estas son de una naturaleza diferente. Cada término adicional en la función de costo tiene un factor de peso, que se utiliza para ajustar la importancia, o el costo de ese elemento, de acuerdo a los objetivos de control. Estos parámetros tienen que ser diseñados adecuadamente, para lograr un comportamiento adecuado de la estrategia de control.

Desafortunadamente, no hay métodos teóricos, analíticos, o numéricos, para ajustar estos parámetros y actualmente se determinan en base a procedimientos empíricos. No obstante, esto no ha impedido que MPC se aplique con éxito en varios convertidores de potencia.

\section{FunCión DE COSTO EN MPC}

El control MPC resuelve un problema de optimización, caracterizado por el error entre la variable predicha y la referencia aplicada al sistema [2]. La función de costo representa el comportamiento deseado, por tanto el modelo predictivo calcula la combinación óptima que minimiza esta función. Una función de costo puede ser muy compleja, dependiendo de las variables u objetivos de control que se consideran. Sin embargo, estas variables dependen solo de la aplicación

TABLA I

OBJETIVOS DE CONTROL BÁSICOS EN MPC.

\begin{tabular}{cc}
\hline Variable & Función de Costo \\
\hline Corriente & $g_{i}=\left|i_{\alpha}^{*}-i_{\alpha}^{p}\right|+\left|i_{\beta}^{*}-i_{\beta}^{p}\right|$ \\
Voltaje & $g_{v}=\left|v_{o \alpha}^{*}-v_{o \alpha}^{p}\right|+\left|v_{o \beta}^{*}-v_{o \beta}^{p}\right|$ \\
Torque & $g_{T}=\left|T_{e}^{*}-T_{e}^{p}\right|$ \\
Flujo & $g_{\Psi}=\left|\Psi_{s}^{*}-\Psi_{s}^{p}\right|$ \\
Potencia activa & $g_{P}=\left|P^{*}-P^{p}\right|$ \\
Potencia reactiva & $g_{Q}=\left|Q^{*}-Q^{p}\right|$ \\
Frecuencia de conmutación & $g_{n}=n_{c}$ \\
\hline
\end{tabular}


en la que intervienen. En la Tabla I se recogen algunos de los objetivos de control más utilizados y la función de costo asociada de acuerdo a la literatura. Los índices $* \mathrm{y}$ $p$ representan las variables de referencias y las variables predichas respectivamente. Entre ellos, destacan las funciones que consideran la corriente $(i)$, el voltaje $(v)$, el torque $(T)$ o la potencia $(P, Q)$ como objetivos de control. Por otro lado, objetivos como la minimización del voltaje de rizado, la frecuencia de conmutación $(n)$, o la potencia, pueden lograrse incluyendo variables específicas en la función de costo [10], [11].

Los términos más comunes en una función de costo son los que se muestran en la ecuación (1). Estos términos se pueden expresar de manera general como el error entre la variable predicha y su referencia correspondiente.

$$
g=\left\|x^{*}-x^{p}\right\|
$$

donde $x^{*}$ es el valor de referencia y $x^{p}$ es el valor predicho de la variable controlada para un estado de conmutación determinado. La norma $\|\cdot\|$ es una medida de distancia entre los valores de referencia y los valores predichos y por lo general, se puede implementar para un período de muestreo como: i) un valor absoluto representado en (2), ii) un valor cuadrado mostrado en (3), o iii) un valor integral del error representado en (4).

$$
\begin{gathered}
g=\left|x^{*}-x^{p}\right| \\
g=\left(x^{*}-x^{p}\right)^{2} \\
g=\left|\int_{k}^{k+1}\left(x^{*}(t)-x^{p}(t)\right) d t\right|
\end{gathered}
$$

Cuando la función de costo considera solamente un error, las alternativas para calcular el error absoluto y el error cuadrado ofrecen resultados similares. En cambio, si presenta dos, o más términos, los mejores resultados los ofrece la función de costo cuadrada [9]. El error cuadrado presenta mejor seguimiento de la referencia cuando se agregan términos adicionales a la función de costo. La función de costo considera la trayectoria de las variables entre los intervalos de tiempo $t_{k}$ y $t_{(k+1)}$, conduciendo a la minimización del error medio, lo que implica un seguimiento más preciso.

En la Tabla II muestra algunos ejemplos de funciones de costo implementadas en la literatura. En la función de costo cuadrático, los pesos proporcionan una compensación entre el error de seguimiento y el esfuerzo realizado por la estrategia de control. Algunas de las funciones de costo, o combinaciones más utilizadas en MPC, se describen en las siguientes investigaciones:

- suma ponderada de error de seguimiento y esfuerzo de control [12], [13];

- función de costo cuadrático para el seguimiento del error y el esfuerzo de controlador [13], [14];

- suma del costo de la energía y el costo de la demanda [15];

- suma del error de seguimiento [16];
- potencia integrada o consumo de energía [14];

- costo de operación [17];

La mayoría de los investigadores han intentado minimizar la suma ponderada del error de seguimiento y el esfuerzo del controlador. En cambio, otros solo han minimizado la suma del error de seguimiento, o error instantáneo (aumentando como resultado el costo de operación). La función de costo dinámico también se puede utilizar para colocar diferentes pesos en la función objetivo y con ello favorecer la estrategia de control.

Debido a las diferencias en las variables y magnitudes de los elementos dentro de la función de costo, se introducen inconvenientes que necesitan ser abordados de manera eficiente. La solución a este problema ha sido abordada de diferentes maneras, aunque algunas de ellas coinciden en incluir coeficientes de peso o factores de ponderación $\lambda$, para cada término de la función de costo:

$$
g=\lambda_{x}\left\|x^{*}-x^{p}\right\|+\lambda_{y}\left\|y^{*}-y^{P}\right\|+\ldots+\lambda_{Z}\left\|Z^{*}-Z^{p}\right\|
$$

Dependiendo de la naturaleza de los diferentes términos involucrados en la formulación de la función costo, se pueden clasificar en diferentes grupos, algunos de los cuales se describen y ejemplifican a continuación.

\section{A. Funciones de costo sin factores de peso}

Cuando las funciones de costo solo controlan una variable (o las componentes de una variable), se denominan funciones de costo sin factores de peso. De esta manera, un elemento no requiere factores de ponderación, y resulta una función más simple.

Algunos ejemplos representativos de este tipo de función se obtienen para: el control predictivo de corriente de un inversor fuente de voltaje (VSI), el control predictivo de potencia de un rectificador AFE (Active Front End), control predictivo de voltaje en un sistema de alimentación ininterrumpida (UPS), control predictivo de corriente con frecuencia de conmutación fija y control predictivo de corriente en inversores multifásicos, entre otros [9]. Estas funciones de costo se resumen en la Tabla III.

Todos los términos en estas funciones de costo están compuestos por variables de la misma naturaleza (unidad y

TABLA II

EJEMPLOS DE FUNCIONES DE COSTO DE ACUERDO A LA LITERATURA [2]

\begin{tabular}{ccc}
\hline Variables & Descripción & Valor \\
\hline & {$[2]$} & $g=|q|+\lambda\left|i_{L}^{*}-i_{L}^{p}\right|$ \\
CSC-AFE & {$[18]$} & $g=(q)^{2}+\lambda\left(i_{L}^{*}-i_{L}^{p}\right)^{2}$ \\
& {$[9]$} & $g=\left|i_{k}^{*}-i_{k}^{p}\right|$ \\
VSC-AFE & {$[2]$} & $g=\left|i_{k}^{*}-i_{k}^{p}\right|+\lambda_{n} n_{c}$ \\
& {$[19]$} & $g=\left(i_{k}^{*}-i_{k}^{p}\right)^{2}$ \\
Motores & {$[20]$} & $g=\left(P^{*}-P^{p}\right)^{2}+\left(Q^{*}-Q^{p}\right)^{2}$ \\
VSC-UPS & {$[21]$} & $g=\left(T^{*}-T^{p}\right)^{2}+\left(\psi^{*}-\psi^{p}\right)^{2}$ \\
Statcom & {$[22]$} & $g=\left(v_{o}^{*}-v_{o}^{p}\right)^{2}$ \\
Convertidores & {$[23]$} & $g=\left(i_{k}^{*}-i_{k}^{p}\right)^{2}$ \\
Matriciales & {$[24]$} & $g=\left|\left(i_{L}^{*}-i_{L}^{p}\right)\right|^{2}+\lambda\left|Q^{*}-Q^{p}\right|$ \\
HVDC & {$[25]$} & $g=\left(i_{L}^{*}-i_{L}^{p}\right)^{2}+\lambda\left(Q^{*}-Q^{p}\right)^{2}$ \\
& {$[26]$} & $g=g_{1}+g_{2}+g_{3}$ \\
& & $g=\left|i_{j k}^{*}-i_{j k}^{p}\right|$ \\
\hline
\end{tabular}


TABLA III

Funciones DE COSTO SIN FACTORES DE PESO [27]

\begin{tabular}{ll}
\hline Aplicación & Función de costo \\
\hline Control predictivo de corriente de un VSI & $\left|i_{\alpha}^{*}-i_{\alpha}^{p}\right|+\left|i_{\beta}^{*}-i_{\beta}^{p}\right|$ \\
Control predictivo de potencia de un AFE & $\left|Q^{p}\right|+\left|P^{*}-P^{p}\right|$ \\
Control predictivo de voltaje UPS & $\left(v_{c \alpha}^{*}-v_{c \alpha}^{p}\right)^{2}+\left(v_{c \beta}^{*}-v_{c \beta}^{p}\right)^{2}$ \\
Control predictivo de corriente impuesta en VSI & $\left|F\left(i_{\alpha}^{*}-i_{\alpha}^{p}\right)\right|+\left|F\left(i_{\beta}^{*}-i_{\beta}^{p}\right)\right|$ \\
Control de corriente en inversores multifásicos VSI & $\left|i_{\alpha}^{*}-i_{\alpha}^{p}\right|+\left|i_{\beta}^{*}-i_{\beta}^{p}\right|+\left|i_{x}^{*}-i_{x}^{p}\right|+\left|i_{y}^{*}-i_{y}^{p}\right|$ \\
\hline
\end{tabular}

magnitud). Además, algunos de ellos son una descomposición de un solo vector en dos, o más componentes, por lo tanto, no hay factores de peso, o no es necesario realizar un proceso de ajuste. Sin embargo, en ocasiones controlar una sola variable no es suficiente y hay que recurrir a otras estrategias, como el diseño de las funciones de costo con términos secundarios.

\section{B. Funciones de costo con términos secundarios}

En investigaciones recientes, se han definido además de los objetivos principales de la función de costo, otras restricciones $\mathrm{u}$ objetivos secundarios, que pueden mejorar el rendimiento del controlador. En este caso, la función de costo contiene términos primarios y secundarios, donde la importancia de los términos secundarios depende de las necesidades específicas de la aplicación. Algunos ejemplos de estas funciones se muestran en la Tabla IV y son: control predictivo de corriente con reducción de la frecuencia de conmutación [8], control predictivo de corriente con reducción de tensión en modo común (para evitar daños en motores eléctricos [24]), y control predictivo de corriente con reducción de potencia reactiva (para mejorar la calidad de la potencia [24]).

La importancia del segundo término dependerá de las necesidades específicas de la aplicación, e impondrá una relación con el control primario. En cada función de costo se incluye un factor de peso $\lambda$, relacionado con el término secundario correspondiente. Por lo tanto, la relación de compromiso se puede ver como un ajuste del factor de peso en la función de costo implementada.

\section{Funciones de costo con términos igualmente importantes}

Cuando las variables a controlar son más de una y cada una de ellas tiene simultáneamente la misma importancia, estamos en presencia de un sistema de control con términos igualmente importantes. En este caso, el trabajo de los factores de peso es compensar la diferencia entre cada una de las variables. Tal es el caso del control de torque y control de flujo de una máquina de inducción, donde ambas variables deben controlarse con precisión para obtener el rendimiento adecuado del sistema [28]. Algunos ejemplos de este tipo de funciones pueden ser resumido en la Tabla V, la cual presenta dos ejemplos de aplicación y sus funciones de costos.

Como se puede apreciar en la literatura, la selección adecuada de la función de costo y el ajuste de los factores de pesos no es una tarea sencilla. En ella intervienen una serie de factores que dificultan su aplicabilidad en sistemas de control predictivo.

\section{Ajuste De los factores De Peso}

A continuación, se describen algunos de los elementos más importantes para ajustar los factores de peso en la función de costo y se muestran algunos resultados. Este procedimiento de ajuste varía según los términos presentes en la función de costo. De esta manera, se utilizarán dos clasificaciones para diferenciarlas.

\section{A. Ajuste de las funciones de costo con términos secundarios}

El ajuste del factor de peso en este caso, se realiza forzando los factores de pesos a un valor cero $(\lambda=0)$. Por lo tanto, el primer paso, es convertir la función de costo con términos secundarios en una función de costo sin factores de peso. Las funciones con estas características son mostradas en la Tabla IV.

El segundo paso es establecer una medida que se utilizará para evaluar el rendimiento alcanzado por el factor de peso. En todos los casos, un valor debe ser relacionado con la variable primaria, y se denomina error actual, mientras por otro lado, es necesario un segundo valor, que será utilizado como referencia. Una vez que se definen las medidas hay que evaluar el comportamiento del sistema con simulaciones que comienzan con $\lambda=0$ y se van aumentando gradualmente hasta que $\lambda=1$ [9]. De esta manera, se detendrá el incremento de $\lambda$ cuando el término secundario alcance el valor deseado para la aplicación específica, o hasta que la variable primaria exceda los valores permisibles. Luego se grafican los resultados y se selecciona un valor de $\lambda$ que cumpla con los requisitos del sistema para ambas variables [27].

En la Tabla VI se muestra el comportamiento del controlador predictivo en un NPC, cuando los valores de $\lambda$ son modificados. En este caso, se definen dos objetivos de control, determinados por la corriente de salida y el voltaje en los capacitores del enlace DC. Como se puede apreciar, el incremento del valor de $\lambda$ implica al aumento del error de predicción de corriente y la minimización del error del control de voltaje en los capacitores.

1) Reducción de la frecuencia de conmutación: este es uno de los ejemplos en los cuales se aplica el ajuste de la función con términos secundarios. Por ejemplo, en la ecuación (6) se presenta un esquema de control predictivo de corriente, para un inversor trifásico VSI, con reducción de la frecuencia de conmutación. La función de costo presenta un término para el seguimiento de la referencia de corriente y un término secundario para la reducción de la frecuencia de conmutación. 
TABLA IV

FUNCIONES DE COSTO CON TÉRMINOS SECUNDARIOS [27]

\begin{tabular}{ll}
\hline Aplicación & Función de costo \\
\hline Control predictivo de corriente con reducción de la frecuencia de conmutación & $\left|i_{\alpha}^{*}-i_{\alpha}^{p}\right|+\left|i_{\beta}^{*}-i_{\beta}^{p}\right|+\lambda_{s w} \eta_{s w}^{p}$ \\
Control predictivo de corriente con reducción de voltaje. & $\left|i_{\alpha}^{*}-i_{\alpha}^{p}\right|+\left|i_{\beta}^{*}-i_{\beta}^{p}\right|+\lambda_{c m}\left|V_{c m}^{p}\right|$ \\
Control predictivo de corriente con reducción de potencia reactiva & $\left|i_{\alpha}^{*}-i_{\alpha}^{p}\right|+\left|i_{\beta}^{*}-i_{\beta}^{p}\right|+\lambda_{Q}\left|Q^{p}\right|$ \\
\hline
\end{tabular}

TABLA V

FUNCIONES DE COSTO CON TÉRMINOS IGUALMENTE IMPORTANTES

\begin{tabular}{ll}
\hline Aplicación & Función de costo \\
\hline Torque y control de flujo & $\frac{1}{T_{c n}^{2}}\left(T_{c}^{*}-T_{c}^{p}\right)^{2}+\frac{\lambda_{\psi}}{\psi_{s n}^{2}}\left(\left|\psi_{s}\right|^{*}-\left|\psi_{s}^{p}\right|\right)^{2}$ \\
Balance del voltaje en el capacitor & $\frac{1}{i_{s n}}\left[\left|i_{\alpha}^{*}-i_{\alpha}^{p}\right|+\left|i_{\beta}^{*}-i_{\beta}^{p}\right|\right]+\frac{\lambda_{\Delta V}}{V_{c n}}\left|\Delta V_{c}^{p}\right|$ \\
\hline
\end{tabular}

$$
g=\left|i_{\alpha}^{*}-i_{\alpha}^{p}\right|+\left|i_{\beta}^{*}-i_{\beta}^{p}\right|+\lambda_{s w} \eta_{s w}^{p}
$$

El término secundario $\eta_{s w}^{p}$ corresponde al número previsto de cambios ocurridos al pasar del estado actual, al estado futuro. Así, al aumentar el factor de peso asociado $\lambda_{s w}$ se espera que este término gane mayor importancia en la función de costo y obligue a una reducción en la frecuencia de conmutación.

Para este caso particular, para mayores valores de $\lambda_{s w}$ se obtiene una reducción en la frecuencia de conmutación, lo que introduce una pequeña distorsión en la corriente de carga. Esto se debe a la reducción en el número de conmutaciones. Por otro lado, si $\lambda_{s w}=0$, se eliminan los factores secundarios en la ecuación, pero es a expensas de un aumento en las pérdidas por conmutación.

2) Reducción de la potencia reactiva de entrada: la ecuación (7) es una de las alternativas más utilizadas para abordar el control predictivo de corriente, con reducción en la potencia reactiva de entrada. Esta función de costo consiste en un término para el control de la corriente de salida, expresado en coordenadas ortogonales, y un término secundario [9].

$$
g=\left|i_{\alpha}^{*}-i_{\alpha}^{p}\right|+\left|i_{\beta}^{*}-i_{\beta}^{p}\right|+\lambda_{Q}\left|Q^{p}\right|
$$

El término adicional en la función de costo es la potencia reactiva de entrada predicha $Q^{p}$, con su correspondiente factor de peso $\lambda_{Q}$. Con el fin de evaluar y seleccionar el valor de $\lambda_{Q}$, las medidas de rendimiento del sistema son: el error de corriente y la magnitud de potencia reactiva de entrada $Q$. Dado que esta función de costo pertenece a la misma clasificación que los dos ejemplos anteriores, se espera un comportamiento similar al aumentar $\lambda_{Q}$.

TABLA VI

CÁlCULO DEL ERROR PARA DIFERENTES VALORES DE $\lambda$

\begin{tabular}{lccc}
\hline Porcentaje de Error & $\lambda=0,05$ & $\lambda=0,4$ & $\lambda=0,95$ \\
\hline Corriente en la fase $a$ & 8.7356 & 9.5504 & 9.8977 \\
Voltaje en los capacitores & 2.6442 & 2.5532 & 2.2574 \\
\hline
\end{tabular}

3) Ajuste de las funciones de costo con términos igualmente importantes: para funciones de costo como las que se ven en la Tabla V, se necesita un procedimiento diferente, debido a que $\lambda$ no puede ser cero. Primero hay que considerar la naturaleza diferente de las variables. Por ejemplo, al controlar el torque $\mathrm{y}$ el flujo, el error del torque puede tener una magnitud distinta a la del flujo, lo que hace que ambas variables no sean igualmente importantes en la función de costo, afectando el rendimiento del sistema. Por este motivo, es necesario normalizar la función de costo, para que todos los términos sean igualmente importantes. Por lo general, un $\lambda$ adecuado se encuentra cerca de 1.

El segundo paso es evaluar el desempeño alcanzado por cada valor del factor de peso. Debe considerarse $\lambda=1$, debido a que $\lambda=0$ es un valor prohibitivo. Cuando se ha alcanzado un pequeño intervalo de factores de peso, donde no hay grandes diferencias en los valores medios obtenidos con el límite superior e inferior del intervalo, se ha obtenido el factor de peso adecuado.

Un ejemplo de este tipo de control se muestra en la Fig. 1. En este caso se controla el voltaje en los condensadores del enlace DC de un convertidor NPC. Como se aprecia el control predictivo cumple la función de predecir y regular ambos valores. De esta manera, ajustando los valores de $\lambda$ adecuadamente dentro de la función de costo, se obtienen errores relativamente bajos para ambos objetivos de control como se aprecia en la Tabla VI.

Por otro lado, el esfuerzo del controlador en un convertidor NPC puede representarse por el cambio en el vector de tensión aplicado a la carga. Esto se implementa como un término adicional en la función de costo que mide la diferencia entre el vector voltaje en la muestra anterior $v(k-1)$ y el vector voltaje que se aplicará $v(k)$. Se considera el control predictivo de corriente y voltaje, cuando la función de costo resultante incluye ambas variables como se expresa en la ecuación (8) y (9).

$$
\begin{gathered}
g=\left\|x^{*}-x^{p}\right\|+\lambda\|v(k-1)-v(k)\| \\
g=\left|i_{\alpha}^{*}-i_{\alpha}^{p}\right|+\left|i_{\beta}^{*}-i_{\beta}^{p}\right|+\lambda\|v(k-1)-v(k)\|
\end{gathered}
$$




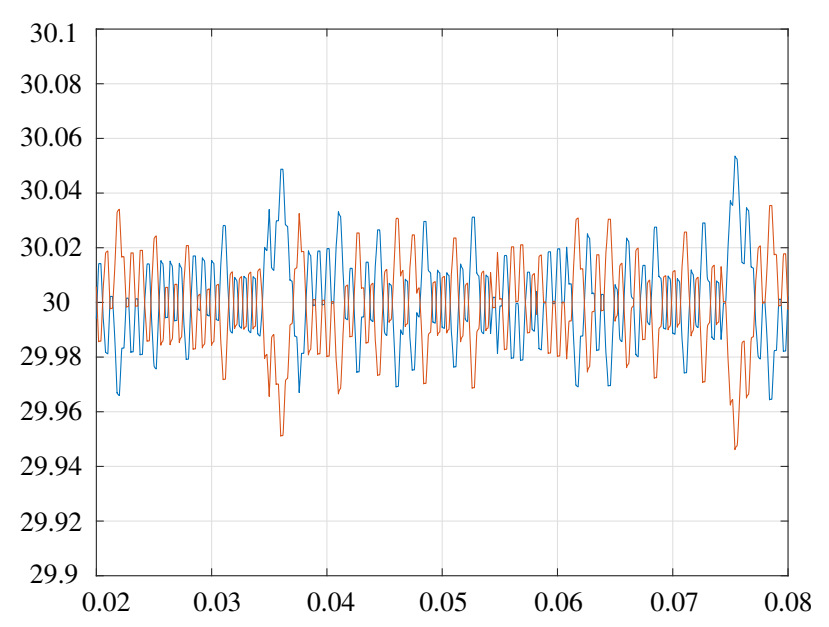

Fig. 1. Control predictivo del voltaje en los condensadores del enlace de DC en un convertidor NPC.

Donde $x$ es la variable controlada y $\lambda$ es un factor de peso que permite ajustar el nivel de compromiso entre el seguimiento de las variables de referencia y el esfuerzo del sistema de control.

El control de un convertidor NPC usando la ecuación (9) se puede apreciar en la Fig. 2. En esta caso, el canal uno $\left(C h_{1}\right)$ muestra la corriente de salida en una de las fases para diferentes valores de $\lambda$. En rojo se muestra $\lambda=0,05$ y en azul $\lambda=0,95$. Además, en la misma figura se muestra el voltaje de salida con los mismos valores del factor de peso anterior en el canal $2\left(C h_{2}\right)$ y el voltaje controlado en uno de los capacitores del enlace DC. Como se puede apreciar en la Fig. 2 y en la Tabla VI, a medida que aumenta el factor de peso $\lambda$, se obtienen mejores resultados en el error del voltaje en los capacitores $\left(C h_{3}\right)$, pero se degrada la corriente de salida en cada una de las fases $\left(C h_{2}\right)$ y por consiguiente el número de conmutaciones para alcanzar el voltaje de salida $\left(C h_{2}\right)$ aumenta, con el incremento del factor de peso asociado al balance del voltaje en los capacitores. Esto demuestra que el aumento del factor de ponderación favorece un objetivo de control, en detrimento de otras variables del sistema. No obstante, si la estrategia de control predictivo es diseñada de manera correcta, el error alcanzado no deberá tener una desviación significativa con el ajuste de los factores de peso en la ecuación. Los resultados para reducir la frecuencia de conmutación se pueden mejorar considerando un error cuadrado para la referencia actual después de los términos de la función de costo.

$$
g=\left(i_{\alpha}^{*}-i_{\alpha}^{p}\right)^{2}+\left(i_{\beta}^{*}-i_{\beta}^{p}\right)^{2}+\lambda\|v(k-1)-v(k)\|
$$

\section{B. Minimización de la frecuencia de conmutación}

Una de las principales medidas del esfuerzo del controlador es la conmutación de los interruptores. Es por ello que es muy importante poder controlar, o limitar el número de conmutaciones de los interruptores que intervienen en un convertidor de potencia, para garantizar mayor estabilidad.

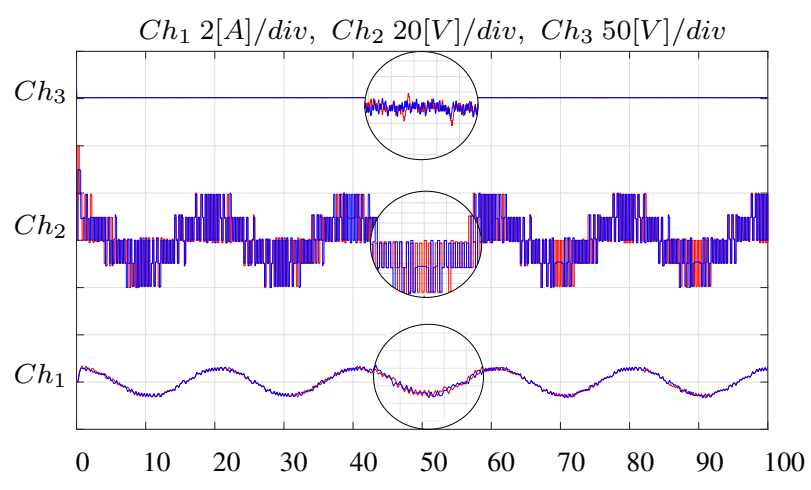

Fig. 2. Control predictivo de voltaje en un capacitor del enlace de DC del convertidor NPC $\left(\mathrm{Ch}_{3}\right)$. Voltaje de salida en una de las fases del convertidor $\left(C h_{2}\right)$. Control predictivo de corriente en una fase $\left(C h_{1}\right)$. El valor rojo representa un factor de ponderación de $0.05 \mathrm{y}$ el valor azul un valor de 0.95 para el control del voltaje de los capacitores de acuerdo a la ecuación (9)

Para considerar directamente la reducción del número de conmutaciones en la función de costo, se puede incluir un término que incluya la cantidad de interruptores que cambian cuando se aplica el estado de conmutación $S(k)$, con respecto a la conmutación aplicada en el estado anterior $S(k-1)$. La función de costo resultante se expresa en (6) y el comportamiento de la frecuencia de conmutación para diferentes valores de factor de ponderación $\lambda_{n}$ indican que esta estrategia mejorará la eficiencia del convertidor. No obstante, al disminuir el número de conmutaciones se puede degradar la calidad del voltaje de salida del convertidor. Sin embargo, al igual que en el caso anterior, un buen diseño de la función de costo y los factores de peso garantizan una baja tasa de error al variar $\lambda$.

\section{CONCLUSIONES}

En esta investigación se presenta el procedimiento para ajustar los factores de peso de la función de costo en un esquema de control predictivo. Se identifican tres tipos de funciones de costo y se describe un procedimiento de trabajo para seleccionar adecuadamente la ideal para nuestros objetivos. De esta manera, la primera etapa del ajuste del factor de peso es identificar el tipo de función de costo que se utilizará en el control. Para funciones de costo con un objetivo de control primario y términos secundarios, el punto de partida es $\lambda=0$ y luego se prueban los incrementos en el valor de $\lambda$ hasta que el comportamiento deseado es obtenido. Para funciones de costo con términos igualmente importantes, la función de costo debe ser normalizada, y luego el factor de peso debe establecerse en $\lambda=1$. Con este valor, el sistema estará controlado y para ajuste más preciso, se pueden usar ligeras variaciones de $\lambda$ alrededor de 1 . Por último, los resultados de las simulaciones nos permitieron validar estos planteamientos y ratificar al control predictivo basado en modelos, como una herramienta muy potente para el control de múltiples objetivos en convertidores de potencia.

\section{Agradecimientos}

Los autores desean agradecer al Programa FONDECYT Regular a través del proyecto 1160690 y a la Beca de Doctorado 
Nacional CONICYT 2019, folio 21190709.

\section{REFERENCIAS}

[1] S. Kwak, U.-C. Moon, and J.-C. Park, "Predictive-control-based direct power control with an adaptive parameter identification technique for improved afe performance," IEEE Transactions on Power Electronics, vol. 29, no. 11, pp. 6178-6187, 2014.

[2] S. Vázquez Pérez, J. Rodríguez, M. Rivera, L. García Franquelo, and M. Norambuena, "Model predictive control for power converters and drives: Advances and trends," IEEE Transactions on Industrial Electronics, 64 (2), 935-947., 2017.

[3] S. Yan, J. Chen, T. Yang, and R. Hui, "Improving the performance of direct power control using duty cycle optimization," IEEE Transactions on Power Electronics, 2018.

[4] J. Sawma, F. Khatounian, E. Monmasson, L. Idkhajine, and R. Ghosn, "Robustness study of a cascaded dual model-predictive control applied to synchronous motors," IEEE Transactions on Industrial Electronics, vol. 66, no. 9, pp. 7219-7228, 2018.

[5] A. Linder, R. Kanchan, P. Stolze, and R. Kennel, Model-based predictive control of electric drives. Cuvillier, 2010.

[6] S. Mariéthoz and M. Morari, "Explicit model-predictive control of a pwm inverter with an lcl filter," IEEE Transactions on Industrial Electronics, vol. 56, no. 2, pp. 389-399, 2009.

[7] S. Vazquez, J. Leon, L. Franquelo, J. Rodriguez, H. A. Young, A. Marquez, and P. Zanchetta, "Model predictive control: A review of its applications in power electronics," IEEE Industrial Electronics Magazine, vol. 8, no. 1, pp. 16-31, 2014.

[8] R. Vargas, P. Cortés, U. Ammann, J. Rodríguez, and J. Pontt, "Predictive control of a three-phase neutral-point-clamped inverter," IEEE Transactions on Industrial Electronics, vol. 54, no. 5, pp. 2697-2705, 2007.

[9] J. Rodriguez and P. Cortes, Predictive control of power converters and electrical drives. John Wiley \& Sons, 2012, vol. 40.

[10] M. Mamdouh and M. A. Abido, "Efficient predictive torque control for induction motor drive," IEEE Transactions on Industrial Electronics, vol. 66, no. 9, pp. 6757-6767, 2018.

[11] D. Zhou, P. Tu, and Y. Tang, "Multivector model predictive power control of three-phase rectifiers with reduced power ripples under nonideal grid conditions," IEEE Transactions on Industrial Electronics, vol. 65, no. 9, pp. 6850-6859, 2018.

[12] J. Rehrl and M. Horn, "Temperature control for hvac systems based on exact linearization and model predictive control," in 2011 IEEE International Conference on Control Applications (CCA). IEEE, 2011, pp. 1119-1124.

[13] P.-D. Moroşan, R. Bourdais, D. Dumur, and J. Buisson, "Building temperature regulation using a distributed model predictive control," Energy and Buildings, vol. 42, no. 9, pp. 1445-1452, 2010.

[14] J. Sirokỳ, F. Oldewurtel, J. Cigler, and S. Prívara, "Experimental analysis of model predictive control for an energy efficient building heating system," Applied energy, vol. 88, no. 9, pp. 3079-3087, 2011.
[15] J. Ma, J. Qin, T. Salsbury, and P. Xu, "Demand reduction in building energy systems based on economic model predictive control," Chemical Engineering Science, vol. 67, no. 1, pp. 92-100, 2012.

[16] J. A. Candanedo and A. K. Athienitis, "Predictive control of radiant floor heating and solar-source heat pump operation in a solar house," HVAC\&R Research, vol. 17, no. 3, pp. 235-256, 2011.

[17] G. P. Henze, D. E. Kalz, S. Liu, and C. Felsmann, "Experimental analysis of model-based predictive optimal control for active and passive building thermal storage inventory," HVAC\&R Research, vol. 11, no. 2, pp. 189$213,2005$.

[18] J. Michalík, Z. Peroutka, and V. Šmídl, "Finite control set mpc of active current-source rectifier with full state space model," in IECON 2015-41st Annual Conference of the IEEE Industrial Electronics Society. IEEE, 2015, pp. 004 121-004 126.

[19] Z. Song, Y. Tian, W. Chen, Z. Zou, and Z. Chen, "Predictive duty cycle control of three-phase active-front-end rectifiers," IEEE Transactions on Power Electronics, vol. 31, no. 1, pp. 698-710, 2015.

[20] S. Vázquez Pérez, A. Márquez, R. Aguilera, D. Quevedo, J. I. León Galván, and L. García Franquelo, "Predictive optimal switching sequence direct power control for grid connected power converters," IEEE Transactions on industrial electronics, 62 (4), 2010-2020, 2015.

[21] P. Karamanakos, P. Stolze, R. M. Kennel, S. Manias, and H. du Toit Mouton, "Variable switching point predictive torque control of induction machines," IEEE journal of emerging and selected topics in power electronics, vol. 2, no. 2, pp. 285-295, 2014.

[22] P. Cortés, G. Ortiz, J. I. Yuz, J. Rodríguez, S. Vazquez, and L. G. Franquelo, "Model predictive control of an inverter with output $l c$ filter for ups applications," IEEE Transactions on Industrial Electronics, vol. 56, no. 6, pp. 1875-1883, 2009.

[23] P. Acuna, L. Moran, M. Rivera, J. Dixon, and J. Rodriguez, "Improved active power filter performance for renewable power generation systems," IEEE transactions on power electronics, vol. 29, no. 2, pp. 687 694, 2013.

[24] R. Vargas, M. Rivera, J. Rodriguez, J. Espinoza, and P. Wheeler, "Predictive torque control with input pf correction applied to an induction machine fed by a matrix converter," in 2008 IEEE Power Electronics Specialists Conference. IEEE, 2008, pp. 9-14.

[25] M. Rivera, C. Rojas, A. Wilson, J. Rodriguez, J. Espinoza, C. Baier, and J. Muñoz, "Review of predictive control methods to improve the input current of an indirect matrix converter," IET Power Electronics, vol. 7 , no. 4, pp. 886-894, 2013.

[26] J. Qin and M. Saeedifard, "Predictive control of a modular multilevel converter for a back-to-back hvdc system," IEEE Transactions on Power delivery, vol. 27, no. 3, pp. 1538-1547, 2012.

[27] P. Cortés, S. Kouro, B. La Rocca, R. Vargas, J. Rodríguez, J. I. León, S. Vazquez, and L. G. Franquelo, "Guidelines for weighting factors design in model predictive control of power converters and drives," in 2009 IEEE International Conference on Industrial Technology. IEEE, 2009, pp. 1-7.

[28] Y. Cho, K.-B. Lee, J.-H. Song, and Y. I. Lee, “Torque-ripple minimization and fast dynamic scheme for torque predictive control of permanentmagnet synchronous motors," IEEE Transactions on Power Electronics, vol. 30, no. 4 , pp. 2182-2190, 2015. 